\title{
SURFACE DIFFUSENESS ANOMALY IN HEAVY-ION FUSION POTENTIALS
}

\author{
K. HAGINO \\ Yukawa Institute for Theoretical Physics, Kyoto Unversity, Kyoto 606-8502, Japan \\ E-mail: hagino@yukawa.kyoto-u.ac.jp \\ M. DASGUPTA, I.I. GONTCHAR, D.J. HINDE, C.R. MORTON, J.O. NEWTON \\ Department of Nuclear Physics, Research School of Physical Sciences and \\ Engineering, Australian National University, Canberra, ACT 0200, Australia
}

\begin{abstract}
Recent high precision experimental data for heavy-ion fusion cross sections at energies in the vicinity of the Coulomb barrier systematically show that a strikingly large surface diffuseness parameter for a Woods-Saxon potential is required in order to fit the data. We discuss possible origins for this anomaly, including the effects of dissipation and the sensitivity of fusion cross sections to the choice of inter-nuclear potential. Our study suggests that the frozen density approximation, which is often used in analyses of heavy-ion reactions, may have to be re-examined for heavy-ion fusion.
\end{abstract}

\section{Introduction}

One of the challenging problems in heavy-ion physics is to reproduce simultaneously experimental data for several processes, such as elastic and inelastic scattering, fusion, and particle transfers, within a unified framework. A coupled-channels approach 13 provides an ideal tool for this purpose. The most important ingredient in the coupled-channels calculations is the internuclear potential. A very popular parametrization for this is the Woods-Saxon form (WS), given by

$$
V_{N}(r)=-V_{0} /\left[1+\exp \left(\left(r-R_{0}\right) / a\right)\right] .
$$

This potential has been used widely in analyses of heavy-ion reactions and has enjoyed success.

For scattering processes, it has heen well accepted that the surface diffuseness parameter $a$ is around $0.63 \mathrm{fm}$. 1.5 In marked contrast, recent high precision experimental data for fusion cross sections, which were measured $6 \mathrm{H}^{\mathrm{B}} \mathrm{B}$ with the aim of extracting experimental fusion barrier distributions 9 , suggest that a much larger diffuseness parameter, ranging between 0.8 and $1.4 \mathrm{fm}$, is needed in order to fit the data This is not only for a particular system, but seems to be a general trend. 怔, 2012

We illustrate this problem in Fig. 1 by comparing experimental data for the ${ }^{16} \mathrm{O}+{ }^{208} \mathrm{~Pb}$ fusion reaction $\mathrm{B}$ with various calculations with the WS po- 


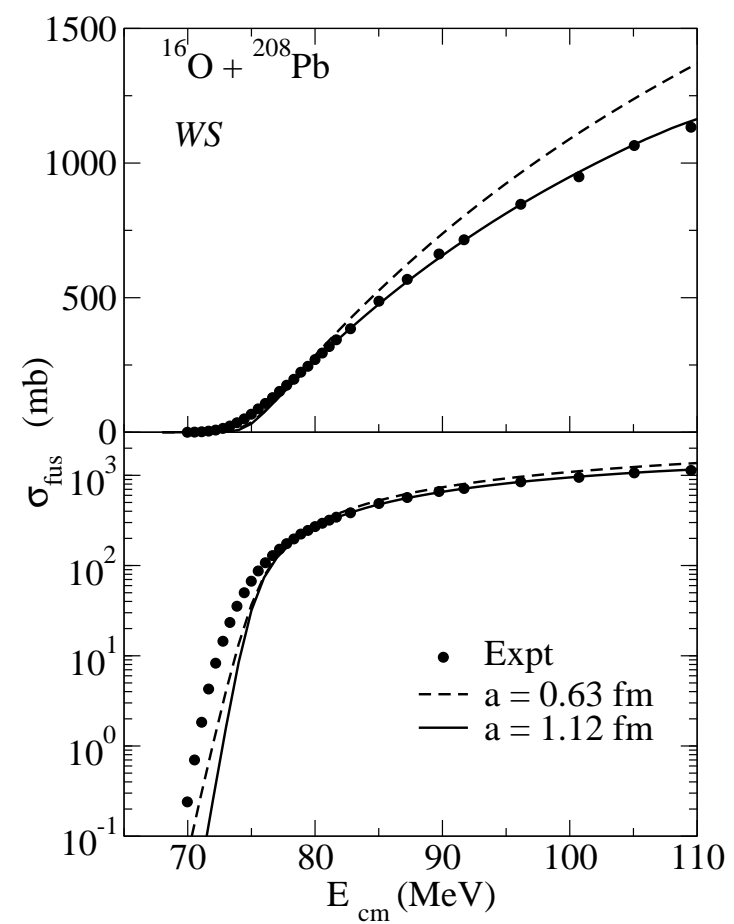

Figure 1: Comparison of potential model with the experimental data for the ${ }^{16} \mathrm{O}+{ }^{208} \mathrm{~Pb}$ fusion reaction. The upper panel is in the linear scale, while the lower panel in the logarithmic scale. The solid and the dashed lines are obtained by setting the surface diffuseness parameter of Woods-Saxon potential to be $a=1.12$ and $a=0.63 \mathrm{fm}$, respectively. The experimental data are taken from Ref. 8.

tential model, which includes the incoming wave boundary condition (see Sec. 2 ) but no channel couplings. The problem is twofold: at energies well above the barrier, where the fusion cross sections $\sigma_{f u s}$ is relatively insensitive to the couplings, a WS potential with a diffuseness $a=0.63 \mathrm{fm}$ significantly overestimates fusion cross sections (dashed line). Changing the $V_{0}$ and $R_{0}$ parameters is not helpful, since it merely leads to an energy shift in the calculated fusion cross sections without significantly changing the energy dependence. On the other hand, a potential with $a=1.12 \mathrm{fm}$ (full line) fits the data well. Below the barrier, the slope of the exponentially falling cross section is given well by the $a=1.12 \mathrm{fm}$ calculation but is not steep enough in that of $a=0.63 \mathrm{fm}$. A better fit to this region would be given by including channel couplings but only if the 
slope were correct. Thus both above and below the barrier the much larger diffuseness parameter $a=1.12 \mathrm{fm}$ is required to match experimental data.

The discrepancy may have a number of causes that include i) channel coupling effects which were not included in the calculations, ii) the deviation of the nuclear potential from the Woods-Saxon parametrization, and iii) friction effects at high angular momenta. Of course, a potential with such a large diffuseness parameter should be regarded as an effective one, which is appropriate only for describing the particular channel. But, it is important to understand the reasons for the large discrepancy in the diffuseness parameters extracted from scattering and from fusion, in order to improve our understanding of the fusion process and to allow reliable predictions of subbarrier fusion cross section.

Among the three possibilities above, the first point was discussed by Esbensen and Back. 13 They showed that the inclusion of collective excitations in both the projectile and the target for the ${ }^{16} \mathrm{O}+{ }^{144} \mathrm{Sm}$ reaction in the coupled-channels calculations significantly improves the fit, leading to a surface diffuseness of $a=0.64 \mathrm{fm}$. However, the effects of channel coupling on the surface diffuseness parameter of the inter-nuclear potential were not shown to be significant for other systems, e.g. ${ }^{16} \mathrm{O}+{ }^{148} \mathrm{Sm}$ reaction, as was discussed in Ref. 14.

In this contribution, we study the other two possible explanations of the surface diffuseness problem. In order to assess the sensitivity of fusion cross sections to the parametrization of the inter-nuclear potential, and to discuss the effects of deviations from the Woods-Saxon shape, we-rill calculate in Sec. 2 fusion cross sections using a double-folding potential 15.16.17, as well as an error function. 11. 18 In Sec. 3, we will discuss the effects of friction on heavy-ion fusion reactions using the critical distance model of Glas and Mosel 19 as well as the surface friction model.20 The summary is given in Sec. 4.

\section{Choice of inter-nuclear potential}

In this section, we use the barrier penetration model to study the sensitivity of fusion cross sections to the choice of inter-nuclear potential. Instead of introducing an imaginary part to the inter-nuclear potential, in the calculations shown below, we use the so-called incoming wave boundary condition. 21 With this boundary condition, it is assumed that there is only an incoming wave inside the Coulomb barrier due to a strong absorption, which should be reasonable for heavy-ion reactions. In this model, fusion cross sections are identified with the absorption cross section. 


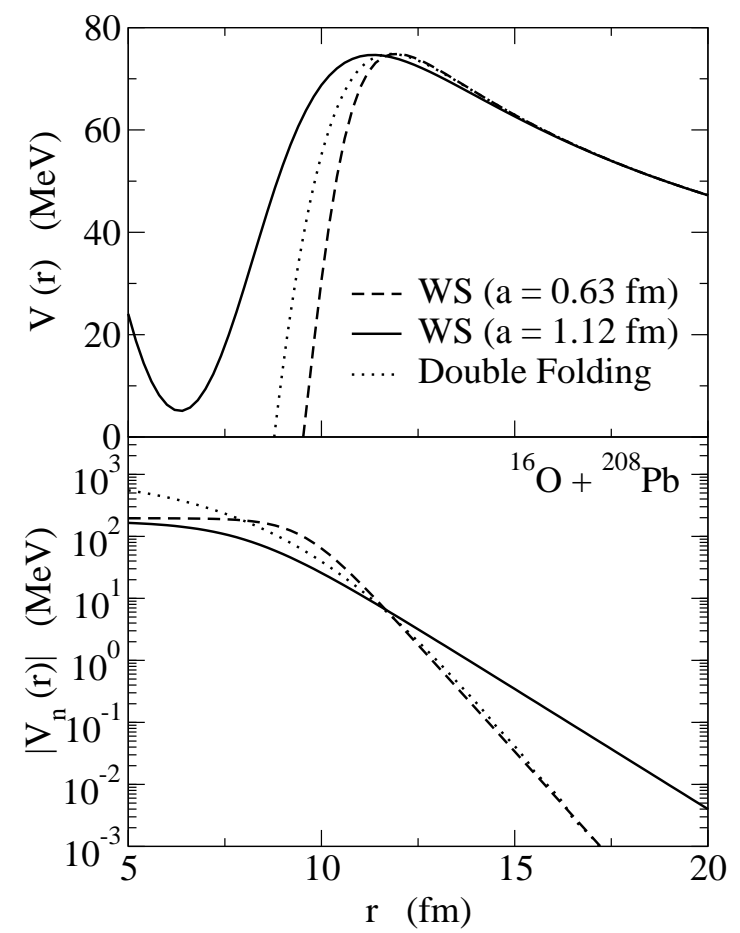

Figure 2: Comparison between the double-folding potential and Woods-Saxon potentials for the ${ }^{16} \mathrm{O}+{ }^{208} \mathrm{~Pb}$ reaction. The upper panel shows the total potential for $s$-wave scattering, while the lower panel shows only the nuclear potential.

\subsection{Double-folding potential}

A double-folding potential 15 , 16 . 17 is constructed by folding an effective nucleonnucleon interaction with the target and the projectile densities:

$$
V_{N}(\boldsymbol{r})=\int d \boldsymbol{r}_{1} d \boldsymbol{r}_{2} v\left(\boldsymbol{r}_{2}-\boldsymbol{r}_{1}-\boldsymbol{r}\right) \rho_{1}\left(\boldsymbol{r}_{1}\right) \rho_{2}\left(\boldsymbol{r}_{2}\right) .
$$

This potential is intimately related to the resonating group method (RGM) 22 and has been widely used in microscopic studies of heavy-ion reactions.

Figure 2 compares the double-folding potential for the ${ }^{16} \mathrm{O}+{ }^{208} \mathrm{~Pb}$ reaction with Woods-Saxon potentials for two different surface diffuseness parameters. For the nucleon-nucleon interaction $v$, we use the density-dependent Michigan three-range Yukawa (DDM3Y1-Paris) interaction 23, together with 


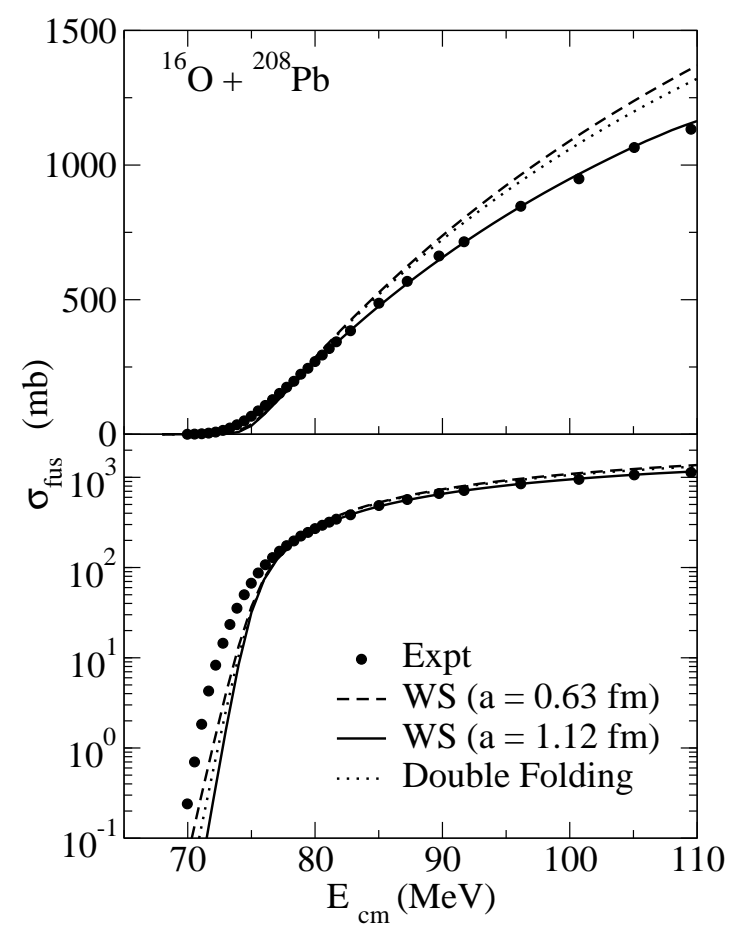

Figure 3: Fusion cross sections obtained with the double-folding potential and their comparison with Woods-Saxon potential for the ${ }^{16} \mathrm{O}+{ }^{208} \mathrm{~Pb}$ reaction.

the zero-range approximation for the exchange contribution (see Ref. 16 for the parameters). We use a Fermi function for the ${ }^{208} \mathrm{~Pb}$ density as is given in Ref. 24, while the same density is used for ${ }^{16} \mathrm{O}$ as that in Ref. 5. The doublefolding potential roughly follows the Woods-Saxon potential with $a=0.63 \mathrm{fm}$, especially at large distances, as would have been expected. It is in general much deeper than the Woods-Saxon potential, and accordingly some deviation from the Woods-Saxon potential is seen in fig.2 at smaller distances. Figure 3 compares fusion cross sections for the double-folding potential with those for the Woods-Saxon potentials. One can see that the double-folding potential leads to similar fusion cross sections to a Woods-Saxon potential with $a=0.63$ $\mathrm{fm}$. The double-folding potential alone, therefore, does not resolve the large diffuseness problem found in heavy-ion subbarrier fusion reactions. 


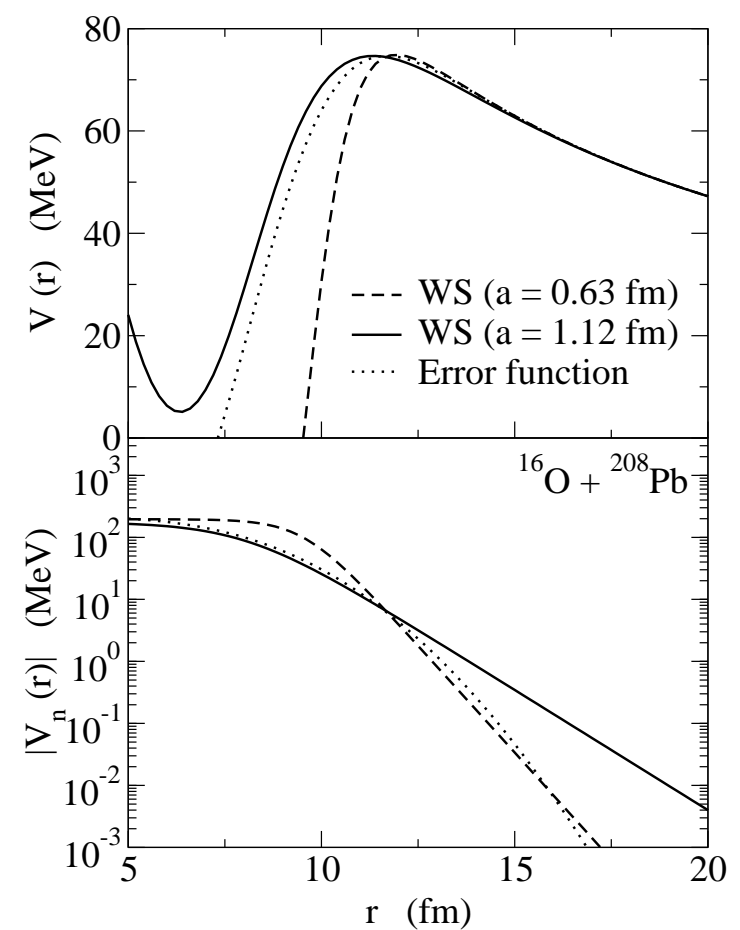

Figure 4: Same as fig. 2, but for an error function potential.

\subsection{Complementary Error Function Potential}

We next consider the error function potential defined by

$$
V_{N}(r)=-\frac{V_{0}}{2} \operatorname{erfc}\left(\left(r-R_{0}\right) / a\right),
$$

where the complementary error function is defined as

$$
\operatorname{erfc}(x)=1-\frac{2}{\sqrt{\pi}} \int_{0}^{x} d t e^{-t^{2}} .
$$

It has been pointed out in Ref. 11 that this potential falls more rapidly than the Woods-Saxon potential with increasing values of $r$ while the curvature of the total potential remains similar. This is demonstrated in Fig. 4, where the error function potential with $V_{0}=220 \mathrm{MeV}, r_{0}=0.92 \mathrm{fm}$, and $a=2.9 \mathrm{fm}$ is compared with the Woods-Saxon potential (note that the $a$ parameter in 


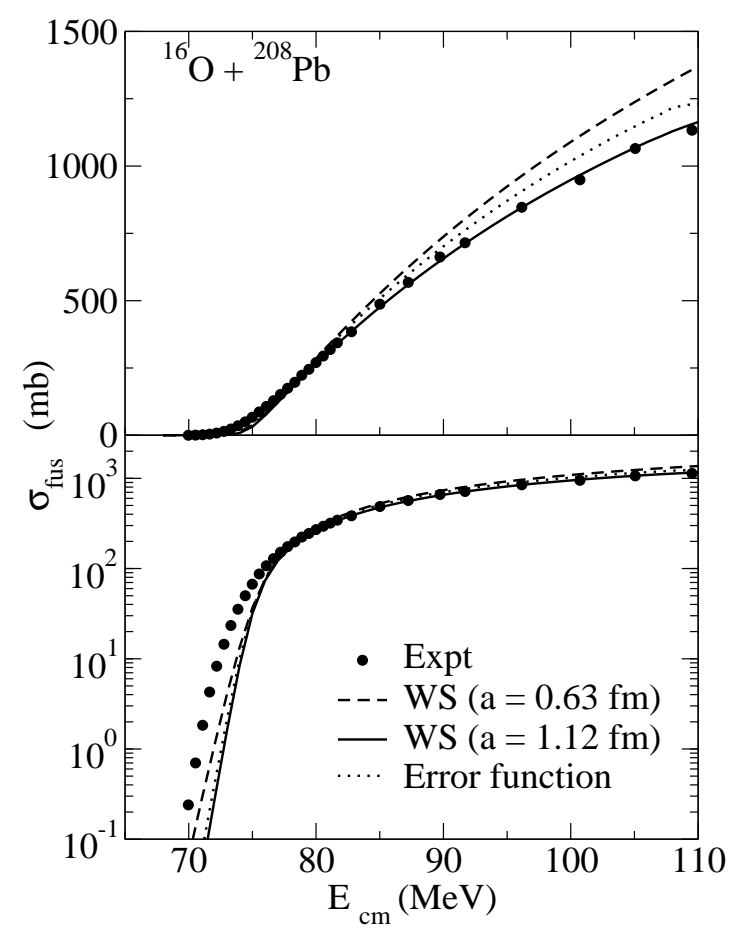

Figure 5: Same as fig. 3, but for an error function potential.

the error function potential (3) does not have a unique correspondence to the surface diffuseness parameter in the Woods-Saxon potential). At distances larger than $r=10.5 \mathrm{fm}$, this potential is very close to the Woods-Saxon potential with $a=0.63 \mathrm{fm}$, while at smaller distances it is similar to the Woods-Saxon potential with $a=1.12 \mathrm{fm}$. As a consequence of the latter, the barrier curvature is nearly the same as that for the Woods-Saxon potential with $a=1.12 \mathrm{fm}$, as can be seen in the upper panel of fig. 4 .

Fusion cross sections obtained with the error function potential are shown in fig. 5. It is interesting to notice that this potential gives fusion cross sections close to the Woods-Saxon potential with $a=1.12 \mathrm{fm}$, thus providing an improved energy dependence of fusion cross sections. As is shown in fig. 4 , this potential is similar to the Woods-Saxon potential with $a=0.63 \mathrm{fm}$ at large distances, and therefore, would lead to similar scattering cross sections. The error function thus provides a promising pqtential which may describe the scattering and fusion processes simultaneously. 11 
The main difference between the error function and the double folding potentials is that the former falls off as a function of inter-nuclear distance in a Gaussian way while the latter falls exponentially. In the folding procedure, it is implicitly assumed that the reaction takes place much faster than the dynamical density evolution. In this approximation, the projectile as well as the target densities remain unchanged even inside the Coulpmb barrier and this leads to an exponential tail of the inter-nuclear potential 25 as a consequence of the fact that the nuclear density behaves exponentially at the surface. This approximation is referred to as the frozen density approximation, and has been well tested in scattering processes. 26 However, if the error function potential reflected the "true "shape of the inter-nuclear potential, its Gaussian tail would suggest a deviation from the frozen density approximation (i.e., an exponential tail) for subbarrier fusion reactions, and effects of dynamical density evolution such as neck formation 27 would have to be taken into account. This is certainly an interesting problem, but is beyond our intention in this contribution and we leave it for future study.

\section{Effects of energy dissipation}

As an alternative origin for the large surface diffuseness problem, in this section we discuss the effects of energy dissipation. A very simple and phenomenological way to mimic friction effects is to use the critical distance model. 1928 (See also Ref. 29). This model assumes that there is a certain distance $R_{c}$ which has to be reached in order for fusion to occur. For each value of $E$, there is a corresponding critical angular momentum $l_{c}$ above which $R_{c}$ is in the classically forbidden region as angular momentum is increased. Only partial waves below $l_{c}$ then contribute to fusion cross sections. Approximating the Coulomb barrier by a parabola, and limiting the partial wave summation up to the critical angular momentum, one-pbtains the expression for the fusion cross section in the Glas-Mosel model 19 :

$$
\sigma_{f u s}(E)=\frac{\hbar \Omega}{2 E} R_{b}^{2} \log \left\{\frac{1+e^{-2 \pi\left(V_{b}-E\right) / \hbar \Omega}}{1+e^{-2 \pi\left(V_{b}-E+\left(E-V_{c}\right) R_{c}^{2} / R_{b}^{2}\right) / \hbar \Omega}}\right\},
$$

where $\Omega, R_{b}$ and $V_{b}$ are the curvature of the Coulomb barrier, the barrier position, and the barrier height, respectively. $V_{c}$ is the value of the total potential at $r=R_{c}$. The critical angular momentum is given by

$$
E=V_{c}+\frac{l_{c}\left(l_{c}+1\right) \hbar^{2}}{2 \mu R_{c}^{2}} .
$$

Note that the Wong formula 30 for the fusion cross section can be recovered by setting $R_{c}$ to be infinity, that is equivalent to not restricting the partial wave 


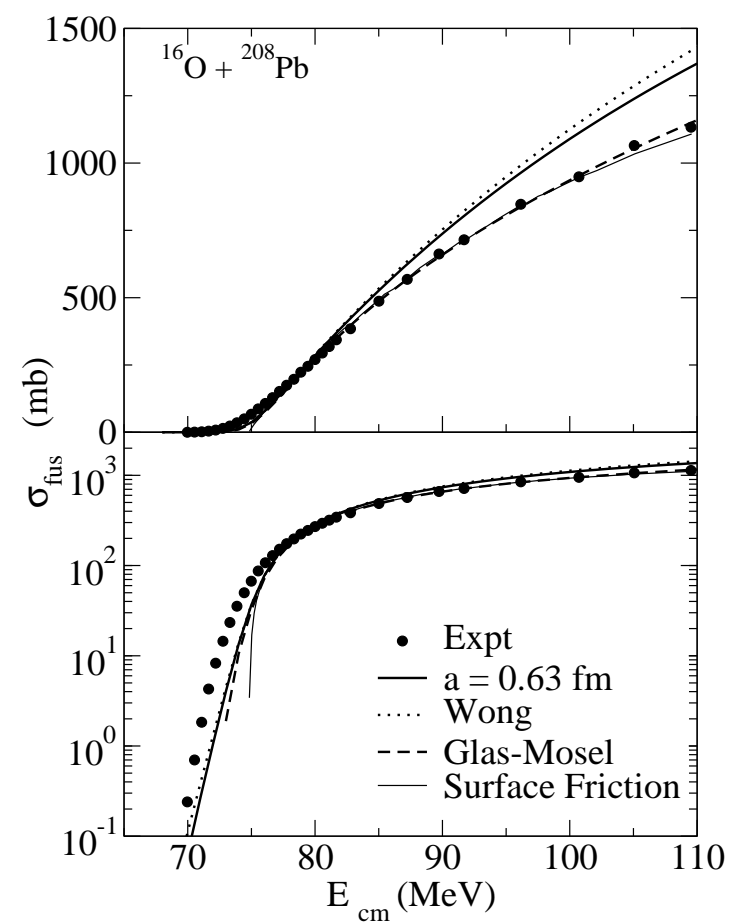

Figure 6: Fusion cross sections calculated with several models. The solid line is the prediction of the barrier penetration model which uses the Woods-Saxon potential with $a=0.63 \mathrm{fm}$, while the dashed line is the result of the Glas-Mosel model. The parabolic approximation to the barrier penetration model is shown by the dotted line as a comparison. The thin solid line is the result of the surface friction model.

summation in computing fusion cross sections.

Figure 6 shows the results of analyses based on the Glas-Mosel model for the ${ }^{16} \mathrm{O}+{ }^{208} \mathrm{~Pb}$ reaction. We first examine the validity of the parabolic approximation used in the Glas-Mosel model. The solid line is the result of the WS potential model with $a=0.63 \mathrm{fm}$. If one approximates this potential by a parabola, the barrier parameters are $R_{b}=11.94 \mathrm{fm}, V_{b}=74.87 \mathrm{MeV}$, and $\hbar \Omega=5.00 \mathrm{MeV}$. The dotted line shows the fusion cross sections obtained with the Wong formula, which is based upon the parabolic approximation. Comparison between the solid and the dotted lines shows that the parabolic approximation works reasonably well in the energy range shown in the figure, although agreement is not perfect.

We now vary $R_{c}$ and $V_{c}$ independently to fit the experimental data, whilst 
retaining the barrier parameters $R_{b}, V_{b}$, and $\hbar \Omega$. The dashed line is for $R_{c}=10.39 \mathrm{fm}$ and $V_{c}=72.4 \mathrm{MeV}$. Although the value of the critical distance $R_{c}$ is larger than the systematics found in Ref. 19, this calculation reproduces the experimental data well at energies above the barrier, and the slope of fusion cross sections below the barrier is very similar to the prediction of the WS potential model with $a=1.12 \mathrm{fm}$. Astonishingly, they are almost indistinguishable if they are plotted in the same figure at this scale.

A more physical but still classical approach to incorporating dissipation is the surface friction model of Fröbrich.20 This model uses a single-folding potential, and friction coefficients which are propotional to the square of the first derivative of the nuclear potential. With slight adjustment of the internuclear potential, and a changed tangential friction, quite good reproduction of the data was obtained (see thin solid line).

The surface-friction model is a macroscopic phenomenological model, and does not consider any particular origin of dissipation. One of the main origins would be inelastic excitations and/or particle transfer. 31 However, for the reaction ${ }^{16} \mathrm{O}+{ }^{208} \mathrm{~Pb}$, the effects of channel coupling are relatively weak due to the double closed shell structure of the projectile and the target. 8 Also it has been pointed out in Ref. 32 that energy dissipation due to particle transfer is largely compensated with the change of potential due to the polarization and thus the Coulomb barrier is reached almost without friction. Therefore neither collective excitations nor particle transfers would lead to the large dissipation effects, at least at the energies we are interested in. The only process which can be attributed to the origin of the dissipation would be a dynamical change in density of the reactants in the region where they overlap. This study again suggests a need to go beyond the frozen density approximation.

\section{Summary}

We studied possible origins of the recent finding that the experimental data for subbarrier fusion cross sections systematically favour a much larger surface diffuseness parameter for a Woods-Saxon form of inter-nuclear potential than the commonly accepted value of $a \sim 0.63 \mathrm{fm}$. An obvious possibility would be that the inter-nuclear potential may deviate from the Woods-Saxon potential. We found that the double-folding potential roughly follows a Woods-Saxon potential with $a=0.63 \mathrm{fm}$, and thus does not account for the large surface diffuseness parameter for subbarrier fusion. On the other hand, parameters could be adjusted for a complementary error function potential so that it looked like a WS with a large surface diffuseness at smaller distances while keeping $a \sim 0.63 \mathrm{fm}$ at the surface. The error function, therefore, provides a promising 
potential which could simultaneously describe fusion and scattering. It falls off in a Gaussian way with increasing inter-nuclear distance whereas a WS potential falls off exponentially. The exponential fall-off is a consequence of the frozen density approximation. Hence, if the error function potential expresses the "true "shape of the inter-nuclear potential, this may suggest a dynamical evolution of density during fusion. As an alternative approach to the problem, we also examined the dissipation effects using the phenomenological critical distance model of Glas and Mosel as well as the surface friction model. The high energy data could be equally well reproduced both by these models and by using the WS potential with a large diffuseness parameter. The fact that the data could be explained both by changes in the potential, and the incorporation of friction, indicates that it may not be a simple matter to disentangle these two effects. We argue that the dynamical density change during the fusion process is one of the main origins of dissipation. This again suggests a deviation from the frozen density approximation, and thus a transition from the sudden to the adiabatic potentials. 33 In this connection, we would like to point out that in the adiabatic picture the inertia parameter may deviate from the reduced mass inside the barrier, as was discussed long ago by Mosel. 34 Apparently, dynamical calculations are needed in order to draw a more conclusive picture

for the surface diffuseness problem. Such calculations are now in progress, and we will report them in future publications.

\section{References}

1. K. Hagino, N. Rowley, and A.T. Kruppa, Comp. Phys. Comm. 123, 143 (1999).

2. I.J. Thompson, Comp. Phys. Rep. 7, 167 (1988).

3. J. Raynal, Phys. Rev. C 23, 2571 (1981).

4. P.R. Christensen and A. Winther, Phys. Lett. B 65, 19 (1976).

5. C.P. Silva et al.,Nucl. Phys. A 679, 287 (2001); L.C. Chamon et al., Nucl. Phys. A 597, 253 (1996).

6. M. Dasgupta, D.J. Hinde, N. Rowley, and A.M. Stefanini, Annu. Rev. Nucl. Part. Sci. 48, 401 (1998).

7. J.R. Leigh, M. Dasgupta, D.J. Hinde, J.C. Mein, C.R. Morton, R.C. Lemmon, J.P. Lestone, J.O. Newton, and N. Rowley, Phys. Rev. C 52, 3151 (1995).

8. C.R. Morton, A.C. Berriman, M. Dasgupta, D.J. Hinde, J.O. Newton, K. Hagino, and I.J. Thompson, Phys. Rev. C 60, 044608 (1999).

9. N. Rowley, G.R. Satchler, and P.H. Stelson, Phys. Lett. B 254, 25 (1991). 
10. C.R. Morton, A.C. Berriman, R.D. Butt, M. Dasgupta, D.J. Hinde, A. Godley, J.O. Newton, and K. Hagino, Phys. Rev. C 64, 034604 (2001).

11. J.O. Newton, C.R. Morton, M. Dasgupta, J.R. Leigh, J.C. Mein, D.J. Hinde, H. Timmers, and K. Hagino, Phys. Rev. C, in press (2001).

12. A.M. Sefanini, L. Corradi, A.M. Vinodkumar, Y. Feng, F. Scarlassara, G. Montagnoli, S. Beghini, and M. Bisogno, Phys. Rev. C 62, 021601 (2001).

13. H. Esbensen and B. Back, Phys. Rev. C 54, 3109 (1996).

14. K. Hagino, S. Kuyucak, and N. Takigawa, Phys. Rev. C 57, 1349 (1998).

15. G.R. Satchler and W.G. Love, Phys. Rep. 55, 183 (1979).

16. M.E. Brandan and G.R. Satchler, Phys. Rep. 285, 143 (1997).

17. D.T. Khoa and G.R. Satchler, Nucl. Phys. A 668, 3 (2000).

18. H. Esbensen, J.Q. Wu, and G.F. Bertsch, Nucl. Phys. A 411, 275 (1983).

19. D. Glas and U. Mosel, Phys. Rev. C 10, 2620 (1974); Nucl. Phys. A 237, 429 (1975).

20. P. Fröbrich, Phys. Rep. 116, 337 (1984).

21. S. Landowne and S.C. Pieper, Phys. Rev. C 29, 1352 (1984).

22. H. Horiuchi, Nucl. Phys. A 522, 257c (1991); see also Sec. 4.3.2 of Ref. 16.

23. D.T. Khoa and W. von Oertzen, Phys. Lett. B 342, 6 (1995).

24. G.R. Satchler, Nucl. Phys. A 579, 241 (1994).

25. Ö. Akyüz and A. Winther, in Nuclear Structure and Heavy-Ion Collisions, Proceedings of the International School of Physics "Enrico Fermi, "Course LXXVII, Varenna, 1979, edited by R.A. Broglia et al. (NorthHolland, Oxford, 1981).

26. C. Ngo, B. Tamain, J. Galin, M. Beiner, and R.J. Lombard, Nucl. Phys. A 240, 353 (1975).

27. C.E. Aguiar, V.C. Barbosa, L.F. Canto, and R. Donangelo, Nucl. Phys. A 472, 571 (1987).

28. J. Galin, D. Guerreau, M. Lefort, and X. Tarrago, Phys. Rev. C 9, 1018 (1974).

29. P. Fröbrich, Phys. Rev. C 39, 2085 (1989).

30. C.Y. Wong, Phys. Rev. Lett. 31, 766 (1973).

31. K. Nishinohara and N. Takigawa, Z. Phys. A 324, 139 (1986).

32. A. Winther, Nucl. Phys. A 594, 203 (1995).

33. J.D. Perez, Nucl. Phys. A 191, 19 (1972)

34. U. Mosel, Particles and Nuclei 3, 297 (1972). 